\title{
miR-494 suppresses the progression of breast cancer in vitro by targeting CXCR4 through the Wnt/ $\beta$-catenin signaling pathway
}

\author{
LINGQIN SONG ${ }^{1}$, DI LIU $^{1}$, BAOFENG WANG ${ }^{1}$, JIANJUN HE ${ }^{2}$, SHUQUN ZHANG ${ }^{1}$, \\ ZHIJUN DAI $^{1}$, XIAOBIN MA ${ }^{1}$ and XIJING WANG ${ }^{1}$ \\ ${ }^{1}$ Department of Oncology, The Second Affiliated Hospital, Medical School of Xi'an Jiaotong University, \\ Xi'an, Shaanxi 710004; ${ }^{2}$ Department of Surgical Oncology, The First Affiliated Hospital, \\ Medical School of Xi'an Jiaotong University, Xi'an, Shaanxi 710061, P.R. China
}

Received January 29, 2015; Accepted April 22, 2015

DOI: $10.3892 /$ or.2015.3965

\begin{abstract}
Breast cancer is the most common cancer among women with a high mortality worldwide, which is mainly due to tumor invasion and metastasis. Previous studies have reported that microRNA-494 (miR-494) is downregulated in breast cancer cells. The present study investigated the role of miR-494 in the progression of breast cancer and the underlying mechanisms. The levels of miR-494 were analyzed in several breast cancer cell lines by quantitative reverse transcription PCR (qRT-PCR). The miR-494 mRNA levels were significantly lower in the malignant breast cancer cells than the level in the non-malignant normal breast epithelial cells. miR-494 mimic transfection upregulated the expression levels of E-cadherin, yet downregulated N-cadherin, vimentin and $\alpha$-smooth muscle actin ( $\alpha$-SMA) in the breast cancer cells. As expected, the expression of these markers in breast cancer cells transfected with miR-494 inhibitors exhibited the opposite variation trend. MTT and Transwell assays showed that cell proliferation and invasion were both significantly suppressed by miR-494 mimics, and were significantly promoted by miR-494 inhibitors. The protein expression level of chemokine (C-X-C motif) receptor 4 (CXCR4) in the breast cancer cells was significantly inhibited by miR-494 mimics, and enhanced by miR-494 inhibitors. Yet, the mRNA level of CXCR4 was barely affected by miR-494 mimics or inhibitors. Dual-luciferase assay confirmed that miR-494 directly interacted with the 3'-untranslated region of CXCR4 mRNA by dual-luciferase assay. The miR-494 mimics also significantly inhibited the transcription levels of $\beta$-catenin, LEF1, CD44 and cyclin-D1, which was similar to the effect of
\end{abstract}

Correspondence to: Dr Lingqin Song, Department of Oncology, The Second Affiliated Hospital, Medical School of Xi'an Jiaotong University, 157 Xiwu Road, Xi'an, Shaanxi 710004, P.R. China E-mail: qinlingsongxa@163.com

Key words: breast cancer, microRNA-494, CXCR4, Wnt/ $\beta$-catenin, epithelial-to-mesenchymal transition
siRNA targeted to CXCR4. In conclusion, miR-494 suppresses the progression of breast cancer through the Wnt/ $\beta$-catenin signaling pathway, which is mediated by CXCR4.

\section{Introduction}

Breast cancer is the most common cancer among women, with an estimated 1.7 million new cases worldwide in 2012 [International Agency for Research on Cancer (IARC), 2012]. Tumor invasion and metastasis affect more than $90 \%$ of patients with breast cancer and are the main factors that lead to high mortality (1). To date, there has been no significant breakthrough in the control of breast cancer.

Epithelial-to-mesenchymal transition (EMT) is believed to be one of the main factors that promotes breast cancer progression. EMT has been known to impart metastasis and stemness characteristics in several types of cancers, including breast cancer, through inducing cancer cells to change from an epithelial to a mesenchymal phenotype, resulting in cancer cell invasion and metastasis (2-4). Furthermore, EMT also enhanced multidrug resistance by upregulating ATP binding cassette transporters in invasive breast cancer cells (5). The modulation of EMT includes numerous gene regulatory networks, including microRNAs (miRNAs) (6).

miRNAs are small non-coding RNA molecules that inhibit gene expression by interacting preferentially with the 3'-untranslated regions (3'-UTRs) of target mRNAs (7). Recently, a number of miRNAs, such as miR-205, miR-21, miR-30a, miR-96 and miR-107 have been shown to be involved in metastasis and cell proliferation, as well as EMT in breast cancer $(8,9)$. In particular, miR-494 appears to play different roles in various types of tumors. miR-494 was shown to induce tumor necrosis factor-related apoptosis-inducing ligand (TRAIL) resistance in non-small cell lung cancer, and is considered an an oncogenic miRNA mega cluster that regulates the G1/S transition during liver tumorigenesis $(10,11)$. In contrast, miR-494 is downregulated in human cholangiocarcinomas, prostate and breast cancer, and gastric carcinoma, and its reinforcement results in cancer growth inhibition, which indicates that miR-494 acts as an anti-oncogene (12). However, the role and the underlying mechanism of miR-494 in breast cancer are still not fully understood. In the present study, we 
focused on the role of miR-494 in the progression of breast cancer and the related molecular mechanism.

\section{Materials and methods}

Cell lines, culture and treatment. The human breast cancer cell lines MDA-MB-231, MCF-7, MDA-MB-468, MDA-MB-435, T47D, BT-474, SK-BR-3, ZR-75-30 and human normal breast epithelial cell line MCF-10A were all obtained from the American Type Culture Collection (ATCC; Manassas, VA, USA). The 293T cells were also purchased from ATCC. The breast cancer cells were cultured in Dulbecco's modified Eagle's medium (DMEM) supplemented with $10 \%$ fetal bovine serum (both from Gibco, Carlsbad, CA, USA), penicillin $(100 \mathrm{U} / \mathrm{ml})$ and streptomycin $(100 \mu \mathrm{g} / \mathrm{ml})$ (Enpromise, China). MCF-10A cells were cultured in Mammary Epithelial Basal Medium (Lonza, Walkersville, MD, USA). Cells were incubated at $37^{\circ} \mathrm{C}$ in a humidified chamber supplemented with $5 \% \mathrm{CO}_{2}$.

Upregulation and downregulation of miR-494. miR-494 (mature sequence, UGAAACAUACACGGGAAACCUC) mimic, inhibitor, miRNA mimic negative control and miRNA inhibitor negative control were purchased from Sigma-Aldrich Inc. (St. Louis, MO, USA). The MDA-MB-231 cells were cultured to $\sim 30-40 \%$ confluency in 6-well plates, and were transfected with miR-494 mimics, miRNA mimic negative control, miR-494 inhibitors or miRNA inhibitor negative control using Lipofectamine 2000 (Invitrogen, Carlsbad, CA, USA), in accordance with the manufacturer's instructions. After $48 \mathrm{~h}$ of incubation, cells were harvested for further analysis. All transfections were performed in triplicate.

CXCR4 siRNA transfection. For targeted knockdown of chemokine (C-X-C motif) receptor 4 (CXCR4), two siRNAs targeted to CXCR4 were designed according to a previous study (13) and synthesized by Genetimes Technology (Shanghai, China). The sequence were: 5'-UAAAAUCUUCCUGCCCCCdTdT-3' and 5'-GGAAGCUGUUGGCUGAAAAdTdT-3'. The non-specific control siRNA was provided by Genetimes Technology. For transfection, 5x10 4 MDA-MB-231 cells seeded in each cell of 24-well microplates were transfected with siRNAs at a final concentration of $100 \mathrm{nmol} / 1$ using Lipofectamine 2000.

Quantitative reverse transcrption-polymerase chain reaction. Total RNA from cultured cells was isolated using TRIzol reagent (Life Technologies, Inc., Rockville, MD, USA) according to the manufacturer's instructions. Reverse transcription was performed on $1 \mu \mathrm{g}$ of total RNA from each sample using oligo(dT) primers and 200 units of SuperScript II (Life Technologies, Inc.) for extension. Quantitative real-time RT-PCR (qRT-PCR) analysis of CXCR4, $\beta$-catenin, lymphoid enhancer-binding factor 1 (LEF1), cyclin-D1, CD44, E-cadherin, $\mathrm{N}$-cadherin, vimentin, $\alpha$-smooth muscle actin $(\alpha$-SMA) and $\beta$-actin was carried out using the primers shown in Table I with the SYBR-Green Master Mix (Takara, Dalian, China) according to the manufacturer's instructions. PCR amplifications were performed using the LightCycler 2.0 (Roche, Basel, Switzerland) with the following amplification
Table I. Primers for the quantitative RT-PCR.

\begin{tabular}{ll}
\hline Name & \multicolumn{1}{c}{ Sequence } \\
\hline miR-494 & \\
Sense & 5'-CATAGCCCGTGAAACATACACG-3' \\
Antisense & 5'-GTGCAGGGTCCGAGGT-3' \\
CXCR4 & \\
Sense & 5'-GGTGGTCTATGTTGGCGTCT-3' \\
Antisense & 5'-TGGAGTGTGACAGCTTGGAG-3' \\
CTNNB1 & \\
Sense & 5'-GCCAGAGCCAACGTCAAGCATCTC-3' \\
Antisense & 5'-GGCAAAGTGTCCAAAACAAAGCCC-3' \\
LEF1 & \\
Sense & 5'-TGCTTGCCTGATGACTACCTG-3' \\
Antisense & 5'-TGAGCACATTTCGGCAATAG-3' \\
CD44 & \\
Sense & 5'-CTCGATGAGGAAGGGTTTGA-3' \\
Antisense & 5'-GCAGGAGGAAGAAGAAGCAC-3' \\
CCND1 & \\
Sense & 5'-ATGTCCCTCGCAAATTGAAG-3' \\
Antisense & 5'-TCAAAATCGAGATCCCCTTG-3' \\
E-cadherin & \\
Sense & 5'-TGGAATCCAAGCAGAATTGC-3' \\
Antisense & 5'-TATGTGGCAATGCGTTCTCTATCCA-3' \\
N-cadherin & \\
Sense & 5'-TGTTGCTGCAGAAAACCAAG-3' \\
Antisense & 5'-TTTCACAAGTCTCGGCCTCT-3' \\
Vimentin & \\
Antisense & 5'-CAAGATCATTGCTCCTCCTGAGCGCA-3' \\
Antisense & 5'-CCCAAGCTTCTCTTCTTGCAAAGATTCCAC-3' \\
A-SMA & \\
Antisense & 5'-TCCAGAGCGACATAGCACAG-3' \\
\hline & \\
5'-CCGAGATCTCACCGACTACC-3'
\end{tabular}

CXCR4, chemokine (C-X-C motif) receptor $4 \alpha$-SMA, $\alpha$-smooth muscle actin

conditions: $95^{\circ} \mathrm{C}$ for $15 \mathrm{sec}, 58^{\circ} \mathrm{C}$ for $1 \mathrm{~min}, 72^{\circ} \mathrm{C}$ for $1 \mathrm{~min}$ and $2 \mathrm{sec}$ for plate reading for 35 cycles. $\beta$-actin was used as the control for normalizing the gene expression. Each measurement was performed in triplicate. Data obtained were calculated according to the $2^{-\Delta \Delta \mathrm{Ct}}$ method (14).

For miR-494 detection, the miR-494-3p was firstly obtained by reverse transcription PCR based on the total RNA using a ReverTra Ace kit (Toyobo Life Science, Shanghai, China). The primer used for reverse transcription PCR was: 5'-GTCGTATCCAGTGCAGGGTCCGAGGTATTCGCACT GGATACGACGAGGTT-3'. The reverse transcription condition was $42^{\circ} \mathrm{C}$ for $30 \mathrm{~min}$ and $95^{\circ} \mathrm{C}$ for $5 \mathrm{~min}$. The qRT-PCR primers for miR-494 are listed in Table I. The amplification conditions were $95^{\circ} \mathrm{C}$ for $10 \mathrm{sec}, 55^{\circ} \mathrm{C}$ for $20 \mathrm{sec}, 72^{\circ} \mathrm{C}$ for $20 \mathrm{sec}$ and $2 \mathrm{sec}$ for plate reading for 35 cycles. 
Western blot analysis. Total proteins were extracted and separated by $12 \%$ SDS-polyacrylamide gel electrophoresis (SDS-PAGE). The bands were then electroblotted onto a nitrocellulose membrane (Amersham, Little Chalfont, UK), followed by blocking non-specific binding using $2 \%$ non-fat dry milk in Tris-buffered saline at room temperature for $2 \mathrm{~h}$. Western blotting was carried out by incubating the membrane with the following antibodies: anti-CXCR4 (1:1,000; Abcam, Cambridge, UK), anti- $\beta$-actin (1:6,000; KangCheng, Beijing, China), anti-E-cadherin (1:800), anti-N-cadherin (1:800), antivimentin $(1: 2,000)$, anti- $\alpha$-SMA (1:800) (all from Proteintech Group), anti- $\beta$-catenin (1:1,000), anti-LEF1 (1:800), anti-CD44 $(1: 800)$ and anti-cyclin-D1 (1:1,000) (all from Cell Signaling Technology, Danvers, MA, USA) antibodies diluted in the blocking buffer overnight at $4^{\circ} \mathrm{C}$. The membrane was then incubated with horseradish peroxidase (HRP)-conjugated secondary antibodies (Santa Cruz Biotechnology, Santa Cruz, CA, USA) for $1 \mathrm{~h}$ at room temperature. 4-Chloro-1-naphthol (4-CN) was used for protein visualization.

Dual-luciferase reporter gene assay. The segments of wild-type 3'-UTR of CXCR4 and mutated 3'-UTR of CXCR4 were obtained according to a previous study (15). The wildtype 3'-UTR of CXCR4 or mutated 3'-UTR of CXCR4 was cloned into the downstream of the luciferase reporter gene in the pGL3 plasmid (Promega, Madison, WI, USA) by XbaI and NotI restriction sites. Wild-type 3'-UTR of CXCR4 or mutated 3'-UTR of CXCR4 cloned in the pGL3 plasmid were co-transfected with the mimic negative control or miR-494 mimics into HEK293T cells (ATCC) using Lipofectamine 2000. After a 48-h incubation, the luciferase activity was measured using the Dual-Luciferase Reporter Assay kit (Promega).

MTT assay. MTT assay was performed to assess cell proliferation. MDA-MB-231 cells were transfected with miR-494 mimics or inhibitors in 96 -well plates $\left(1 \times 10^{4}\right.$ cells/well). After 12,24 and $48 \mathrm{~h}$ of transfection, culture medium was replaced with fresh medium containing MTT (Sangon, Shanghai, China) and incubated with the cells for another $4 \mathrm{~h}$. Then formazan was dissolved in dimethylsulfoxide (DMSO) (150 $\mu \mathrm{l} / \mathrm{well}$; Sigma, St. Louis, MO, USA) for $10 \mathrm{~min}$, and the absorbance value at $550 \mathrm{~nm}$ in each well was measured with a microplate reader (Thermo Fisher Scientific, Waltham, MA, USA).

Matrigel invasion assay. A 24-well permeable support plate (Millipore) were pre-coated with $15 \mu \mathrm{l}$ of Matrigel (BD Biosciences, Bedford, MA, USA). Cells $\left(2 \times 10^{4}\right)$ in $200 \mu \mathrm{l}$ growth media were placed onto the top chamber. Normal culture medium containing $100 \mathrm{ng} / \mathrm{ml} \mathrm{SDF}-1$ was added to the bottom chamber. Plates were incubated for $36 \mathrm{~h}$ at $37^{\circ} \mathrm{C}$. The top of the Matrigel coated permeable support was rubbed using a cotton swab moistened with medium to remove the non-invading cells. The cells on the lower surface of the membrane were fixed with methanol and stained with crystal violet solution. Invaded cells were counted in 10 random fields under a microscope.

Statistical analysis. Unless otherwise stated, all experiments were performed with triplicate samples and repeated at least three times. Results are presented as mean \pm SD. Data are

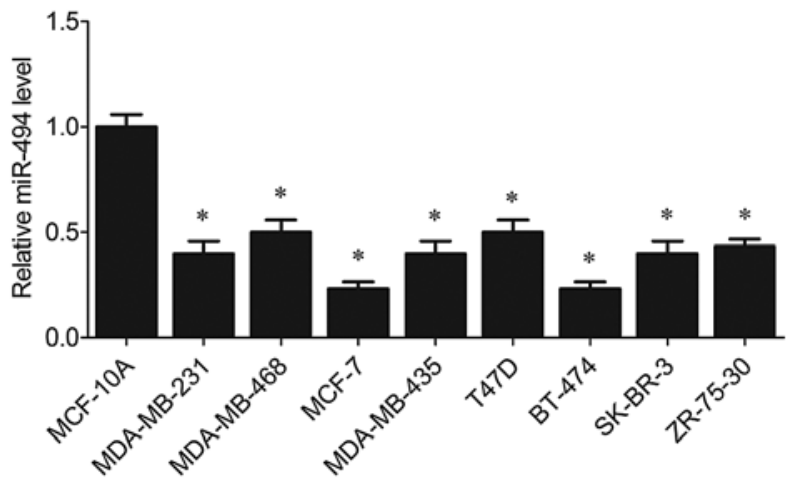

Figure 1. miR-494 expression levels in human breast cancer and human normal beast epithelial cells. The MDA-MB-231, MCF-7, MDA-MB-468, MDA-MB-435, T47D, BT-474, SK-BR-3 and ZR-75-30 are breast cancer cell lines. The human normal breast epithelial cell line MCF-10A was utilized as a control. ${ }^{*} \mathrm{P}<0.05$ compared to the control group.

analyzed by the unpaired Student's t-test. Statistical comparisons between groups were performed using one-way ANOVA followed by the Student's t-test. Analyses were conducted using SPSS 13.0 software. A value of $\mathrm{P}<0.05$ was considered to indicate a statistically significant result.

\section{Results}

miR-494 is downregulated in human breast cancer cell lines. Previous studies demonstrated that miR-494 plays different roles in various types of tumors, either as an oncogenic miRNA in non-small cell lung cancer and liver tumorigenesis $(10,11)$, or as an anti-oncogene in human cholangiocarcinomas, prostate cancer and gastric carcinoma $(12,15,16)$. Therefore, expression of miR-494 was assessed in breast cancer cell lines in the present study. It was revealed by RT-PCR that miR-494 was significantly suppressed in all breast cancer cell lines (MDA-MB-231, MCF-7, MDA-MB-468, MDA-MB-435, T47D, BT-474, SK-BR-3 and ZR-75-30) compared with the level in the non-malignant breast epithelial cell line MCF-10 $(\mathrm{P}<0.05$, Fig. 1).

miR-494 mimics suppress EMT, proliferation and invasion of breast cancer cells. Previous studies have demonstrated that miR-494 is a potential regulatory miRNA in the EMT of cancer, which is the main cause of metastasis of breast cancer. The EMT phenotype is dictated by E-cadherin, $\mathrm{N}$-cadherin, vimentin and $\alpha$-SMA. To investigate the association between the expression of miR-494 and EMT of breast cancer, expression of the EMT markers was detected in the MDA-MB-231 cells transfected with miR-494 mimics or inhibitors. The mRNA level of miR-494 was increased significantly following transfection of the miR-494 mimics in the MDA-MB-231 cells. The reverse effect was shown in cells transfected with the miR-494 inhibitors ( $\mathrm{P}<0.05$, Fig. 2A). RT-PCR analysis demonstrated that miR-494 mimic transfection significantly increased the mRNA levels of E-cadherin and decreased that of $\mathrm{N}$-cadherin, vimentin and $\alpha$-SMA compared to the untreated control cells $(\mathrm{P}<0.05$, Fig. $2 \mathrm{~B})$. As expected, in cells transfected with miR-494 inhibitors, the expression of E-cadherin was significantly decreased, and the expression of 
A

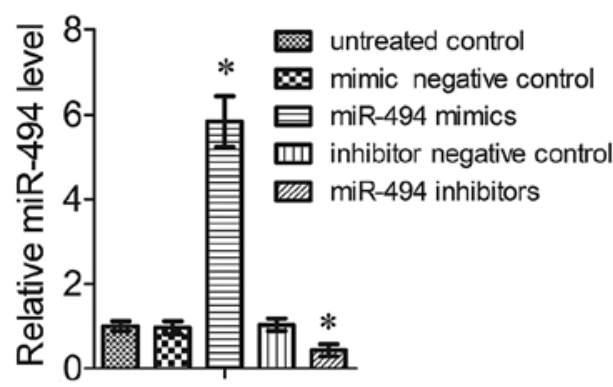

C

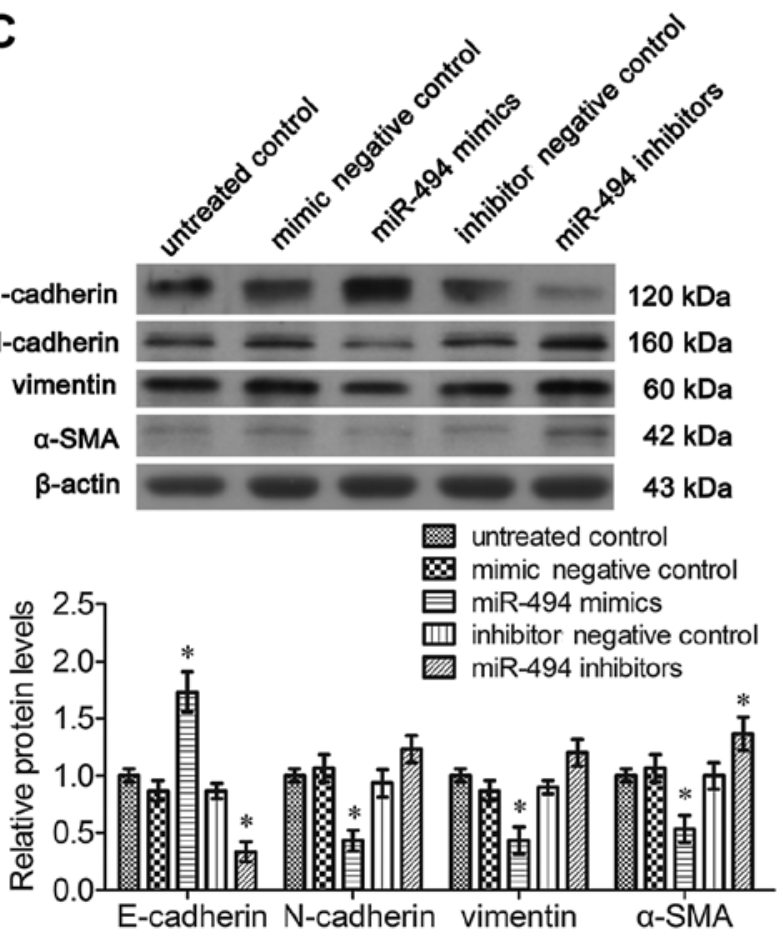

B

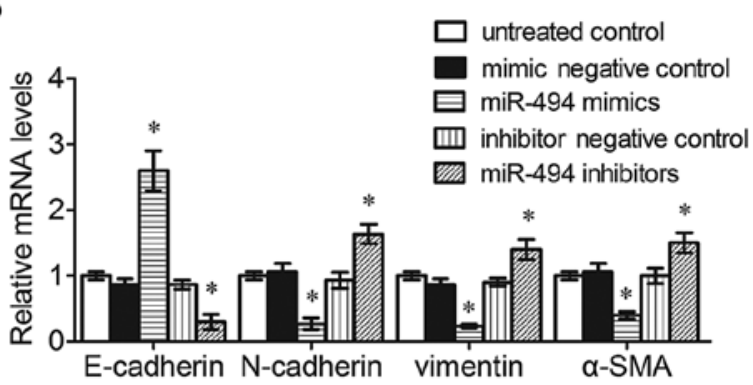

D

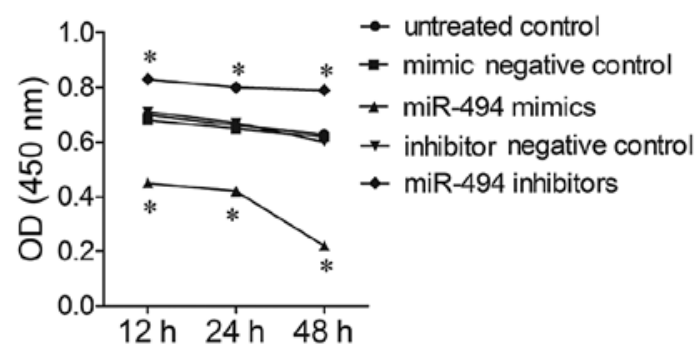

$\mathbf{E}$

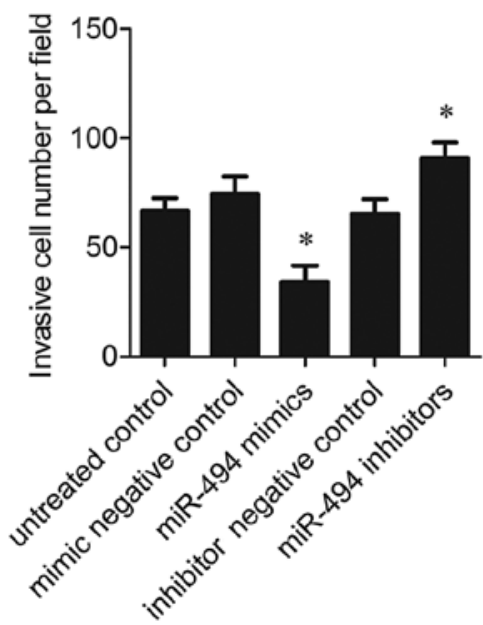

Figure 2. miR-494 suppresses EMT, proliferation and invasion of the MDA-MB-231 cells. (A) MDA-MB-231 cells were transfected with the miR-494 mimics or inhibitors. The miR-494 expression was detected by RT-PCR. Data are presented as relative expression levels. "P $<0.05$ compared to the untreated control cells. (B) mRNA levels of E-cadherin, N-cadherin, vimentin and $\alpha$-SMA in the MDA-MB-231 cells transfected with the miR-494 mimics or inhibitors were detected by RT-PCR. Data are presented as relative expression levels. " $\mathrm{P}<0.05$ compared to the untreated control cells. (C) The protein levels of E-cadherin, $\mathrm{N}$-cadherin, vimentin and $\alpha$-SMA in MDA-MB-231 cells transfected with the miR-494 mimics or inhibitors were detected by western blotting. The bottom graph displays the quantitative result of the protein expression levels. Data are presented as relative expression levels. " $\mathrm{P}<0.05$ compared to the untreated control cells. (D) MTT assay was used to detect the effect of miR-494 on cell proliferation. MDA-MB-231 cells were transfected with the miR-494 mimics or inhibitors. " $\mathrm{P}<0.05$ compared to the untreated control cells. (E) Transwell tumor cell invasion assay. The number of invaded cells was quantified using the mean cell number of 10 random fields (magnification, $x 200$ ). Data are presented as mean $\pm \mathrm{SD}$ of three independent experiments. " $\mathrm{P}<0.05$ compared to the untreated control cells. EMT, epithelial-to-mesenchymal transition; $\alpha$-SMA, $\alpha$-smooth muscle actin.

$\mathrm{N}$-cadherin, vimentin and $\alpha$-SMA was significantly increased compared to the untreated control cells $(\mathrm{P}<0.05$, Fig. 2B). Western blot analysis showed that the expression of these proteins exhibited a similar trend after transfection with the miR-494 mimics or inhibitors, except that N-cadherin and vimentin were not significantly affected by miR-494 inhibitor transfection $(\mathrm{P}<0.05$, Fig. $2 \mathrm{C})$.

MTT assays showed that cells transfected with the miR-494 mimics showed a significant decrease in cell proliferation compared to the untreated controls. Cells transfected with the miR-494 inhibitors showed a small decrease in cell proliferation (Fig. 2D). The Matrigel invasion assay was performed to evaluate the invasive ability of the MDA-MB231 cells transfected with the miR-494 mimics or inhibitors.
The relative invasive cell number was significantly decreased following transfection of the miR-494 mimics, and was markedly increased by miR-494 inhibitor transfection $(\mathrm{P}<0.05$, Fig. 2E).

miR-494 mimics downregulate CXCR4 in breast cancer cells. The mechanism underlying the inhibitory effect of miR-494 on the progression of breast cancer is not fully understood. Previous studies indicated that miR-494 targets CXCR4 to suppress the proliferation, invasion and migration of prostate cancer (15). Therefore, the present study determined the effects of miR-494 on the expression of CXCR4 in the MDA-MB-231 cells. The protein level of CXCR4 was significantly decreased in the cells transfected with the miR494 mimics compared to 

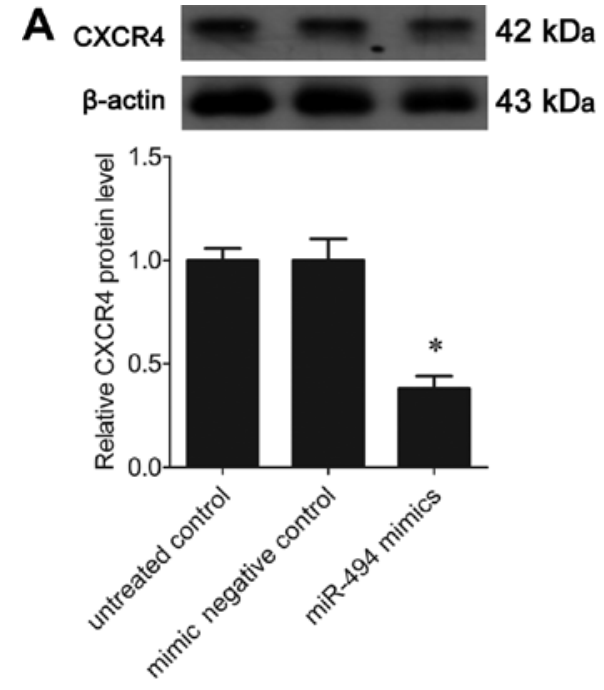

C

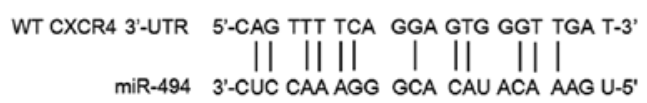

Mut CXCR4 3'-UTR 5'-CAC TAA TGA GGA CAG GCT AGA T-3'

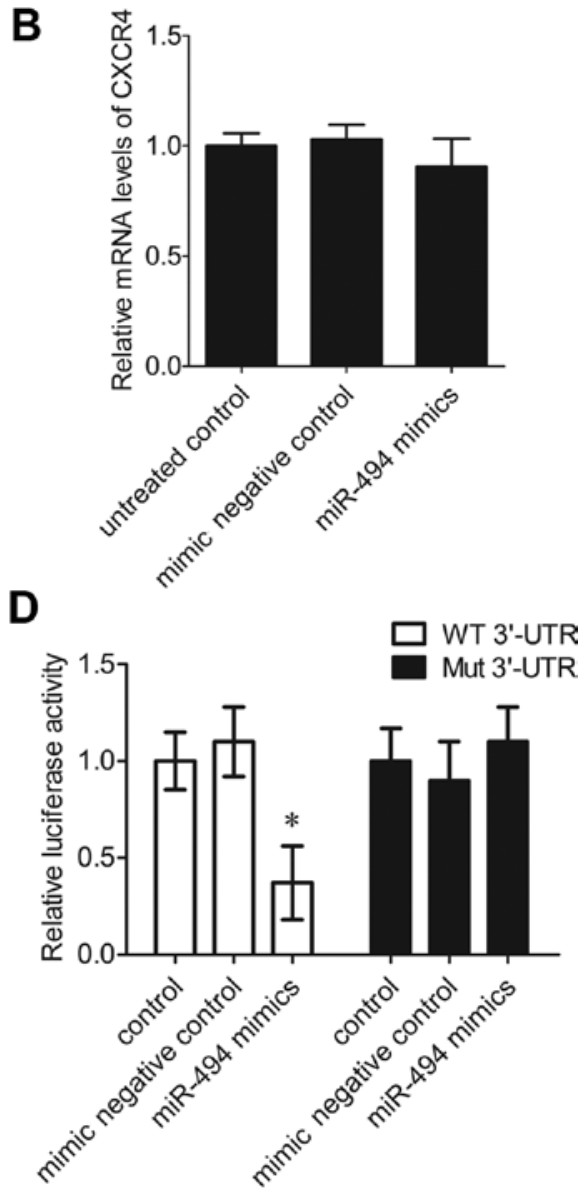

Figure 3. miR-494 mimics downregulate the expression of CXCR4 in the MDA-MB-231 cells. (A) The protein levels of CXCR4 in the MDA-MB-231 cells transfected with the miR-494 mimics or the mimic negative control were detected by western blotting. The bottom graph displays the quantitative result of the protein expression levels. Data are presented as relative expression levels. ${ }^{*} \mathrm{P}<0.05$ compared to the untreated control cells. (B) The mRNA levels of CXCR4 in the MDA-MB-231 cells transfected with the miR-494 mimics or the mimic negative control were detected by RT-PCR. Data are presented as relative expression levels. ${ }^{*} \mathrm{P}<0.05$ compared to the untreated control cells. (C) The predicted binding sequences for CXCR4 3'-UTR by miR-494, and the mutated sequence of CXCR4 3'-UTR. (D) Dual-luciferase reporter gene assays were performed to detect the interaction between 3'-UTR of CXCR4 and miR-494. Wild-type 3'-UTR of CXCR4 or mutated 3'-UTR of CXCR4 constructed in the pGL3 plasmid were co-transfected with the mimic negative control or miR-494 mimics into HEK293T cells. The luciferase activity was measured using a dual-luciferase reporter assay kit. The relative quantification data were normalized to the control group. ${ }^{*} \mathrm{P}<0.05$ compared to the control cells. 3'-UTR, 3'-untranslated region.

the untreated control cells $(\mathrm{P}<0.05$, Fig. $3 \mathrm{~A})$, yet the mRNA level of CXCR4 was not significantly altered (P>0.05, Fig. 3B).

To confirm that miR-494 directly interacts with CXCR4 mRNA, a dual-luciferase reporter assay was performed. Wild-type 3'-UTR of CXCR4 or mutated 3'-UTR of CXCR4 (Fig. 3C) constructed in the pGL3 plasmid were co-transfected with miR-494 mimics into HEK293T cells. Cells only transfected with wild-type or mutated 3'-UTR of CXCR4 were used as a control. The results showed that miR-494 mimics significantly inhibited luciferase activity in the wild-type 3'-UTR of the CXCR4 co-transfected cells as compared with the control group $(\mathrm{P}<0.05$, Fig. 3D). Yet, the reporter constructs with mutated CXCR4 3'-UTR were not affected by miR-494 mimics $(\mathrm{P}<0.05$, Fig. 3D). This revealed that miR-494 binds to CXCR4 mRNA and regulated its translation.

miR-494 suppresses the Wnt/ $\beta$-catenin pathway via targeting $C X C R 4$. Aberrant activation of the canonical Wnt/ $\beta$-catenin pathway plays a critical role in the development of breast cancer. Previous studies have demonstrated that SDF-1/CXCR4 promotes EMT and progression of cancer by activation of the Wnt/ $\beta$-catenin signaling pathway (4). CXCR4 siRNA transfection significantly decreased the mRNA and protein levels of CXCR4 ( $\mathrm{P}<0.05$, Fig. 4A and B). In the MDA-MB231 cells transfected with CXCR4 siRNA, the mRNA and protein levels of $\beta$-catenin, LEF1, CD44 and cyclin-D1 were significantly decreased compared to the untreated control $(\mathrm{P}<0.05$, Fig. 4C and D). Notably, miR-494 mimics also exhibited the same effect on the Wnt $/ \beta$-catenin pathway $(\mathrm{P}<0.05$, Fig. $4 \mathrm{C}$ and D). These data suggest that miR-494 suppresses the Wnt/ $\beta$-catenin pathway via targeting CXCR4.

\section{Discussion}

As the most widespread epithelial tumor among women, breast cancer has been extensively studied in the past decades. Previous studies have focused on the EMT of breast cancer cells, which is the critical factor influencing the invasion and metastasis of tumors, and leads to a poor prognosis. Previous studies have demonstrated that miR-494 is a potential regulatory microRNA in the EMT of cancer. In the present study, significant upregulation of E-cadherin and 


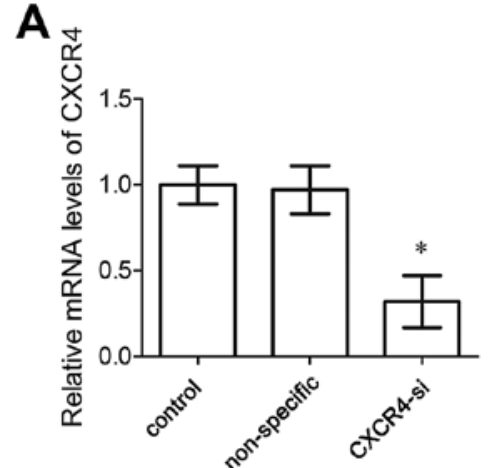

B
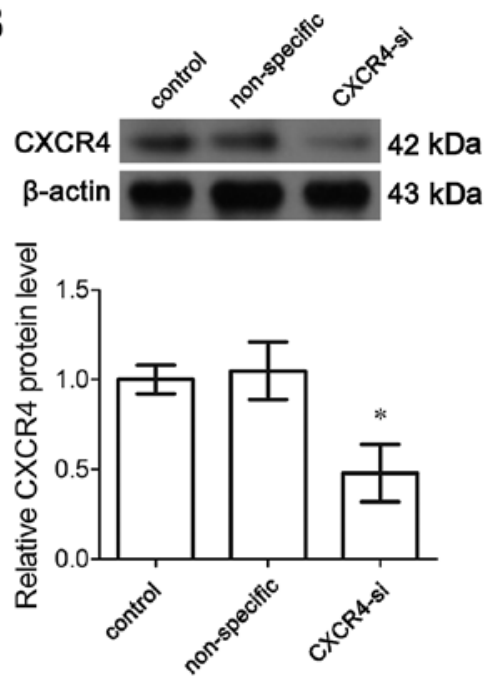

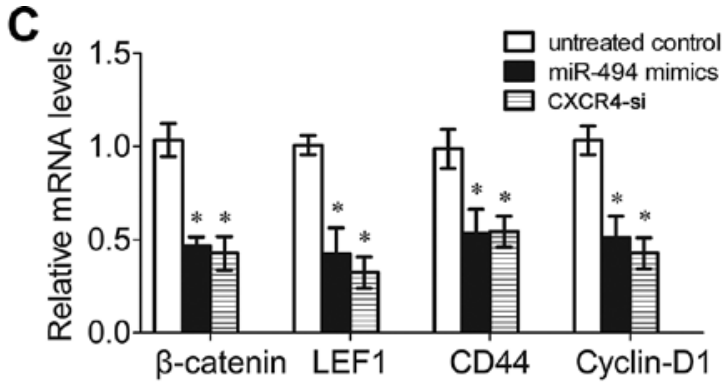

D
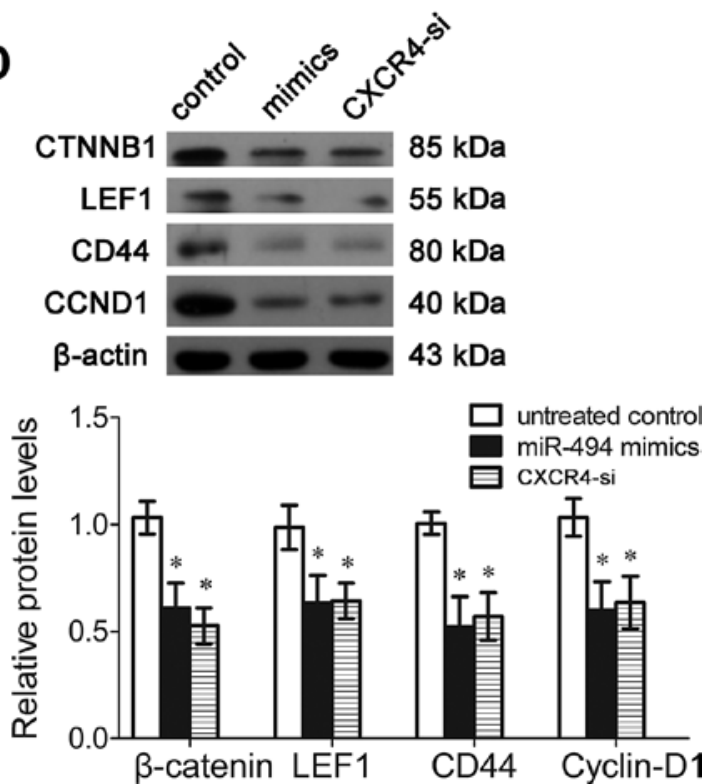

Figure 4. miR-494 mimics suppress the Wnt/ $\beta$-catenin pathway. (A) The mRNA levels of CXCR4 in the MDA-MB-231 cells transfected with the non-specific siRNA or the siRNA specific to CXCR4 were determined by RT-PCR. Data are presented as relative expression levels. * $<<0.05$ compared to the control cells. (B) The protein levels of CXCR4 in the MDA-MB-231 cells transfected with the non-specific siRNA or the siRNA specific to CXCR4 were determined by western blotting. The bottom graph displays the quantitative result of the protein expression levels. Data are presented as relative expression levels. * $\mathrm{P}<0.05$ compared to the control cells. (C) RT-PCR analysis of $\beta$-catenin, LEF1, CD44 and cyclin-D1 mRNA levels showed a significant decrease in the MDA-MB231 cells transfected with the miR-494 mimics or the siRNA specific to CXCR4 compared to the control MDA-MB-231 cells. Data are presented as relative expression levels. ${ }^{*} \mathrm{P}<0.05$ compared to the untreated control group. (D) Western blot analysis showed that in the MDA-MB-231 cells transfected with the miR-494 mimics or the siRNA specific to CXCR4, there was a significant downregulation of $\beta$-catenin, LEF1, CD44 and cyclin-D1. Data are presented as relative expression levels. ${ }^{*} \mathrm{P}<0.05$ compared to the untreated control group. CXCR4, chemokine $(\mathrm{C}-\mathrm{X}-\mathrm{C}$ motif) receptor 4 .

downregulation of $\mathrm{N}$-cadherin, vimentin and $\alpha$-SMA were observed in the miR-494 mimic-transfected cells compared to the control cells, indicating suppression of EMT following miR-494 transfection in breast cancer cells. Notably, the expression levels of E-cadherin, $\mathrm{N}$-cadherin, vimentin and $\alpha$-SMA were not all significantly altered by miR-494 inhibitor transfection. This may be due to the low baseline of miR-494 in breast cancer cells.

CXCR4 is a seven-transmembrane G-protein-coupled receptor for SDF-1. It was reported that SDF-1 signaling through CXCR4 promotes primary tumor growth and metastasis in breast cancer and other malignancies $(17,18)$. It was further found that SDF-1 signaling through CXCR4 promotes metastasis in breast cancer by increasing angiogenesis and by establishing an immunosuppressive tumor microenvironment (18). Research in prostate cancer showed that the constitutive overexpression of miR-494 downregulated the protein level of CXCR4, leading to suppression of proliferation, invasion and migration of prostate cancer (15). However, the effect of miR-494 on the SDF-1/CXCR4 signaling pathway in breast cancer has not yet been studied. In the present study, we found that miR-494 was markedly downregulated in breast cancer cell lines compared to the non-malignant breast epithelial cell line MCF-10A. Furthermore, our data showed that artificial overexpression of miR-494 suppressed the expression of CXCR4 post-transcriptionally, which is consistent with the previous findings in prostate cancer (15). We confirmed that CXCR4 is a target of miR-494. Furthermore, this effect of miR-494 was found to inhibit the invasion, EMT and metastasis of breast cancer cells, which leads to a positive prognosis.

Wingless-related proteins are important regulators of cell proliferation, differentiation and adhesion (19). The Wnt/ $\beta$-catenin signaling pathway plays an important role in the development and regeneration of several tissues by stimulating the growth of stem cells and multipotential progenitors $(20,21)$, particularly in the tumorigenesis of breast cancer by regulating expression of genes essential for mammary stem cell development. Wnt ligands, including WNT3A, WNT4, WNT6, WNT8B, WNT9A and WNT10B were found to be highly expressed in most breast cancer cell lines (22). These ligands play roles through the $\mathrm{Wnt} / \beta$-catenin pathway. The elevated $\beta$-catenin levels in breast cells are supposed to be associated 
with a predisposition to breast cancer $(22,23)$. In the present study, we found that the effect of miR-494 on SDF-1/CXCR4 suppressed the Wnt/ $\beta$-catenin pathway, which may be a potential therapeutic target to prevent breast cancer metastasis and progression.

In conclusion, our findings demonstrated that miR-494 is downregulated in breast cancer cells, and is able to suppress cellular proliferation, migration and invasion through regulation of SDF-1/CXCR4. These results indicate that miR-494 may serve as a potential therapeutic target for breast cancer.

\section{Acknowledgements}

The present study was supported by the Science and Technology Plan Projects of Social Development Plans for Public Relations of Shaanxi Province (no. 2010k01-140).

\section{References}

1. Maruani DM, Spiegel TN, Harris EN, Shachter AS, Unger HA Herrero-González S and Holz MK: Estrogenic regulation of S6K1 expression creates a positive regulatory loop in control of breast cancer cell proliferation. Oncogene 31: 5073-5080, 2012.

2. Malouf GG, Taube JH, Lu Y, Roysarkar T, Panjarian S, Estecio MR, Jelinek J, Yamazaki J, Raynal NJ, Long H, et al: Architecture of epigenetic reprogramming following Twist1-mediated epithelial-mesenchymal transition. Genome Biol 14: R144, 2013

3. Gomes LR, Terra LF, Sogayar MC and Labriola L: Epithelialmesenchymal transition: Implications in cancer progression and metastasis. Curr Pharm Biotechnol 12: 1881-1890, 2011.

4. Hu TH, Yao Y, Yu S, Han LL, Wang WJ, Guo H, Tian T, Ruan ZP, Kang XM, Wang J, et al: SDF-1/CXCR4 promotes epithelialmesenchymal transition and progression of colorectal cancer by activation of the Wnt/ $\beta$-catenin signaling pathway. Cancer Lett 354: 417-426, 2014.

5. Saxena M, Stephens MA, Pathak H and Rangarajan A Transcription factors that mediate epithelial-mesenchymal transition lead to multidrug resistance by upregulating $\mathrm{ABC}$ transporters. Cell Death Dis 2: e179, 2011.

6. Haga CL and Phinney DG: MicroRNAs in the imprinted DLK1-DIO3 region repress the epithelial-to-mesenchymal transition by targeting the TWIST1 protein signaling network. J Biol Chem 287: 42695-42707, 2012

7. Hwang HW, Wentzel EA and Mendell JT: A hexanucleotide element directs microRNA nuclear import. Science 315: 97-100, 2007.

8. Li XH, Qu JQ, Yi H, Zhang PF, Yi HM, Wan XX, He QY, Ye X, Yuan L, Zhu JF, et al: Integrated analysis of differential miRNA and mRNA expression profiles in human radioresistant and radiosensitive nasopharyngeal carcinoma cells. PLoS One 9 : e87767, 2014
9. Zhang C, Li C, Li J, Han J, Shang D, Zhang Y, Zhang W, Yao Q, $\mathrm{Han} \mathrm{L}, \mathrm{Xu} \mathrm{Y}$, et al: Identification of miRNA-mediated core gene module for glioma patient prediction by integrating highthroughput miRNA, mRNA expression and pathway structure. PLoS One 9: e96908, 2014.

10. Romano F, Garancini M, Uggeri F, Degrate L, Nespoli L, Gianotti L, Nespoli A and Uggeri F: Surgical treatment of liver metastases of gastric cancer: State of the art. World J Surg Oncol 10: 157, 2012

11. Lim L, Balakrishnan A, Huskey N, Jones KD, Jodari M, Ng R, Song G, Riordan J, Anderton B, Cheung ST, et al: MicroRNA-494 within an oncogenic microRNA megacluster regulates $\mathrm{G}_{1} / \mathrm{S}$ transition in liver tumorigenesis through suppression of mutated in colorectal cancer. Hepatology 59: 202-215, 2014.

12. Olaru AV, Ghiaur G, Yamanaka S, Luvsanjav D, An F, Popescu I, Alexandrescu S, Allen S, Pawlik TM, Torbenson M, et al: MicroRNA down-regulated in human cholangiocarcinoma control cell cycle through multiple targets involved in the G1/S checkpoint. Hepatology 54: 2089-2098, 2011.

13. Liang Z, Yoon Y, Votaw J, Goodman MM, Williams L and Shim H: Silencing of CXCR4 blocks breast cancer metastasis. Cancer Res 65: 967-971, 2005.

14. Livak KJ and Schmittgen TD: Analysis of relative gene expression data using real-time quantitative PCR and the $2^{-\Delta \Delta C \mathrm{CT}}$ method. Methods 25: 402-408, 2001.

15. Shen PF, Chen XQ, Liao YC, Chen N, Zhou Q, Wei Q, Li X, Wang J and Zeng H: MicroRNA-494-3p targets CXCR4 to suppress the proliferation, invasion, and migration of prostate cancer. Prostate 74: 756-767, 2014

16. Peng C, Zhou K, An S and Yang J: The effect of CCL19/CCR7 on the proliferation and migration of cell in prostate cancer. Tumour Biol 36: 329-335, 2015.

17. Smith MC, Luker KE, Garbow JR, Prior JL, Jackson E, PiwnicaWorms D and Luker GD: CXCR4 regulates growth of both primary and metastatic breast cancer. Cancer Res 64: 8604-8612, 2004.

18. Orimo A, Gupta PB, Sgroi DC, Arenzana-Seisdedos F, Delaunay $T$, Naeem R, Carey VJ, Richardson AL and Weinberg RA: Stromal fibroblasts present in invasive human breast carcinomas promote tumor growth and angiogenesis through elevated SDF-1/CXCL12 secretion. Cell 121: 335-348, 2005.

19. Wielenga VJ, Smits R, Korinek V, Smit L, Kielman M, Fodde R, Clevers $\mathrm{H}$ and Pals ST: Expression of CD44 in Apc and Tcf mutant mice implies regulation by the WNT pathway. Am J Pathol 154: 515-523, 1999.

20. MacDonald BT, Tamai K and He X: Wnt/beta-catenin signaling: Components, mechanisms, and diseases. Dev Cell 17: 9-26, 2009.

21. Nusse R, Fuerer C, Ching W, Harnish K, Logan C, Zeng A, ten Berge D and Kalani Y: Wnt signaling and stem cell control. Cold Spring Harb Symp Quant Biol 73: 59-66, 2008.

22. Benhaj K, Akcali KC and Ozturk M: Redundant expression of canonical Wnt ligands in human breast cancer cell lines. Oncol Rep 15: 701-707, 2006

23. Schlange T, Matsuda Y, Lienhard S, Huber A and Hynes NE: Autocrine WNT signaling contributes to breast cancer cell proliferation via the canonical WNT pathway and EGFR transactivation. Breast Cancer Res 9: R63, 2007. 